\title{
Selective surface smoothening of 3D micro-optical elements
}

\author{
Helmut Schift*a , Nachiappan Chidambaram ${ }^{\mathrm{a}}$, Mirco Altana ${ }^{\mathrm{b}}$, Robert Kirchner ${ }^{\mathrm{a}}$ \\ ${ }^{a}$ Paul Scherrer Institute, Laboratory for Micro- and Nanotechnology, 5232 Villigen PSI, Switzerland \\ ${ }^{\mathrm{b}}$ Heptagon Oy, Moosstrasse 2, 8803 Rüschlikon, Switzerland
}

\begin{abstract}
We have established a non-contact polishing process for thermoplastic, polymeric microlenses and -prisms with dimensions of up to $50 \mu \mathrm{m}$, including sharp convex tips and rims with sub- $\mu \mathrm{m}$ details. The required 3D master structures were fabricated using direct laser-writing lithography with two-photon absorption. Master structures were replicated into poly(methyl methacrylate) through a poly(dimethyl siloxane) intermediate copying step and exposed with $172 \mathrm{~nm}$ UVlight. Due to the reduction of glass transition temperature in a surface-confined layer, roughness in the range of more than $100 \mathrm{~nm}$ could subsequently be smoothed-out to below $10 \mathrm{~nm}$ by annealing the surface by heating.
\end{abstract}

Keywords: Micro-optics, thermoplastic polymers, replication, glass transition temperature, thermal reflow

\section{INTRODUCTION}

The process chain of molding processes leading to polymer components consists of three principal processes: origination, tooling and replication, often followed by post-processing steps such as assembly, integration into frames with a macroscopic interface and packaging [1]. This is valid for replication processes for components with different structure sizes and applications, such as surface topographies for diffractive optical elements (DOE) [2-5] up to high aspect ratio microstructures used as micromechanical elements or microfluidic channels [6-8], and also for micro-optical elements with 3D shapes used in smartphones and sensing devices [9]. These principal processes involve many more process steps, i.e., design, process simulation, stamp copying, transformation into a working tool with appropriate structural resolution, area enlargement and even the integration of mixed micro- and nanostructures with threedimensional (3D) features [10]. What is often omitted is that for specific boundary conditions such as writing time, analysis and testing, different process chains or variants of process chains are needed. It is known that the fabrication of a tool for replication has different requirements than the mass fabrication of individual devices. An expensive origination process may be easily compensated once the replication of a final device is established. Even then, since modern fabrication methods for tools are often still slow and limited in throughput, there are severe restrictions which are difficult to overcome. This is particularly true for novel 3D lithographic tools, which are often developed for research environment only. They are not developed for satisfying demand for large area and complex designs with both, microstructures and sub- $\mu \mathrm{m}$ resolution. Simple examples are optical polymer microlenses which are used in various handheld or mobile devices, including smartphones. They are relatively small in diameter (up to a few $\mathrm{mm}$ ), but since they are often composed of individual lens elements (in a so-called microlens array, MLA) with much smaller dimensions, they have to be processed using lithographic methods that build the lenses out of thin layers (e.g., grayscale electron or laser writing) [11]. However, this often results in steps and roughness, which has adverse effects for optical applications. In a method requiring layering, this can easily be improved by reducing the step height between individual layers (the slicing distance), but consequently results in an enhancement of the number of layers. Therefore not only scaling larger arrays and higher structures will critically enhance the writing time, but also the number of layers necessary to build a 3D topography with surface finish satisfying optical applications. Therefore strategies are needed which enable fast writing but at the same time good surface finish. We have presented a range of possibilities in Ref. [12] and focus here on the selective smoothening by post processing of surface topographies which were optimized for speed but not resolution and roughness.

*helmut.schift@psi.ch; phone +41 56310 2839; fax +41 56310 2646; www.psi.ch/lmn 


\section{SELECTIVE SURFACE SMOOTHENING USING "TASTE"}

If a surface has to be subsequently smoothed out, it must be achieved using a method that only modifies the surface and does not change the underlying layers or overall shape. For this a method known as TASTE was developed (thermally activated surface topography equilibration), which works particularly well with thermoplastic polymers such as poly (methyl methacrylate) (PMMA) [13,14]. It involves selectively changing the material properties of the part of the sample that needs modification, i.e., locally confined in lateral or vertical direction. By using high energy exposure, the polymer is modified by chain scission followed by molecular weight $\left(\mathrm{M}_{\mathrm{w}}\right)$ dependent reflow at elevated temperatures. The original TASTE process did this for an electron beam exposed PMMA film which was modified through its entire height (up to a few $\mu \mathrm{m}$ for $100 \mathrm{keV}$ tools such as the VISTEC EBPG 5000 Plus, Vistec Lithography B.V.) and developed to desired depth resulting in stepped structures. Typical exposure doses applied for grayscale lithography were sufficient for generating a lateral $\mathrm{M}_{\mathrm{w}}$ contrast which was also resulting in a significant difference in glass transition temperature $T_{g}$ from the original polymer enabling selective reflow [14]. This difference was large enough that only the exposed material could flow at a moderate temperature near the $T_{g}$ of the original material, while the unexposed material stayed almost unaltered. For PMMA with a nominal $\mathrm{M}_{\mathrm{w}}$ of about $100 \mathrm{~kg} / \mathrm{mol}$ this was a reduction from $120^{\circ} \mathrm{C}$ down to $90^{\circ} \mathrm{C}$ and even below. Using this, a stepped staircase with steps each $400 \mathrm{~nm}$ high, $500 \mathrm{~nm}$ wide, was transformed into a smooth, linear slope. For surface smoothening of microlenses and -prisms with a roughness of only few $100 \mathrm{~nm}$ height this local modification has to be translated into a depth confined modification. The exposure should cause chain scission of the polymer's backbone, leading to $\mathrm{M}_{\mathrm{w}}$ reduction. Since also small chain fragments are created, which can easily be oxidized and transformed into volatile products, this intentional damage of the polymer also leads to unwanted ablation. As long as this ablation is small enough in comparison to the overall surface topography, it can be neglected. The remaining polymer is modified with different degrees of damage from the surface to the bulk. If this damage is high enough within a layer with a thickness similar to the roughness to be smoothed out, the lateral displacement from material within surface corrugations from small elevated areas to depressions can take place $[15,16]$. The thickness of this modified top layer (skin) does not have to be precisely defined, but should enable to maintain surface topographies at a larger scale than that of the surface corrugations to be eliminated. The test structures we have chosen are microlenses and -prisms with dimensions of $50 \mu \mathrm{m}$ and $20 \mu \mathrm{m}$ in lateral and vertical direction, respectively, including sharp convex tips and rims with sub- $\mu \mathrm{m}$ details. Such structures are particularly delicate because they contain surface slopes with different inclinations, and due to the sequential writing a surface roughness of different degrees

We have found $172 \mathrm{~nm}$ vacuum ultraviolet (VUV) exposure to be the ideal fit for this application [17]. It can be applied in ambient and tools are commercially available for homogeneous, large area exposure. Due to the high absorption in polymeric materials such as PMMA, the depth of damage by exposure can be limited to a few 100's of nm. Although exponential photon intensity decay can be anticipated, parameters could be selected for which the $\mathrm{T}_{\mathrm{g}}$ reduction to values suitable for reflow was confined in a depth of 100 to $500 \mathrm{~nm}$. At the same time, for the doses selected, the ablation of surface molecules during exposure and subsequent heating could be minimized to a few 10's of nm only. This loss of thickness (or possibly also a densification of surface areas which release small chain fragments) is tolerable for most applications where structures of several $\mu \mathrm{m}$ height with surface roughness in the range of $100 \mathrm{~nm}$ need to be treated.

\section{MATERIALS AND METHODS}

\subsection{Replication method}

As described in Ref. [9] and depicted in Fig. 1, the original micro-lens design was written by DLW-2PP with a Nanoscribe Professional GT (Nanoscribe $\mathrm{GmbH}$ ) into a proprietary, negative-tone resist material (IpDip, Nanoscribe $\mathrm{GmbH}$ ) on a fused silica substrate with adhesion promoter (APS1 or OrmoPrime, micro resist technology GmbH). Then it was replicated into UV-curing poly(dimethyl siloxane) (UV-PDMS, micro resist technology $\mathrm{GmbH}$ ) and transferred into PMMA (PMMA120k, micro resist technology GmbH) by thermal nanoimprint lithography (NIL) [18]. The PMMA was spin-coated in 4 iterative steps giving each about $10 \mu \mathrm{m}$ thickness to obtain the required $40 \mu \mathrm{m}$ film thickness on a Borofloat $^{\mathbb{B}}$ glass substrate. Both, the IpDip original on fused silica as well as the PDMS replica on Borofloat ${ }^{\mathbb{B}}$ were treated with an antisticking layer in a chemical vapor deposition process at room temperature following an low power RIE $\mathrm{O}_{2}$ plasma activation with $20 \mathrm{sccm} \mathrm{O}_{2}$ gas flow, 20 mTorr chamber pressure, 20 s plasma time, $20 \mathrm{~W}$ RF power (Plasmalab 80, Oxford Instruments) [19]. The replication into PMMA was performed in a Jenoptik HEX 03 press at 
2.5 $\mathrm{MPa}$ and $180^{\circ} \mathrm{C}$ with a holding time at high pressure of $10 \mathrm{~min}$. Figure 1 shows a schematic of the process chain from origination to surface reflow involving two replication steps.

(a)

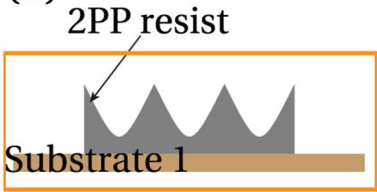

Master structure

(f)

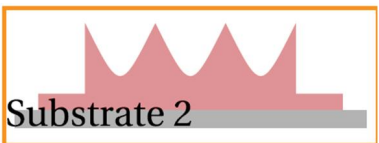

Replica of the master (b)

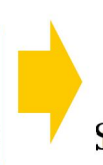

Sulst$$
\text { S }
$$

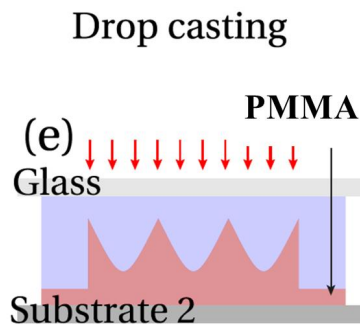

Thermal NIL

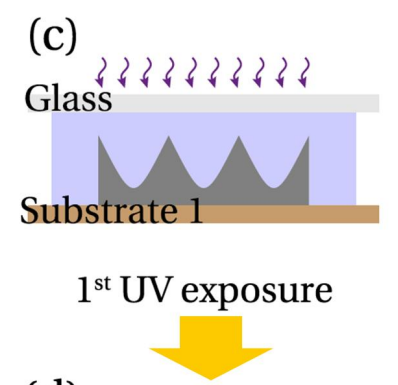

(d) Stamp
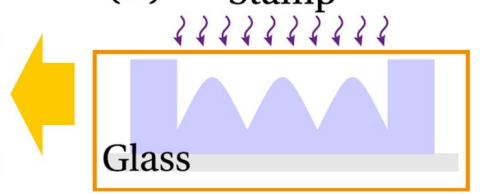

Demolding

$\& 2^{\text {nd }}$ exposure

Figure 1. Schematic of the process flow starting from definition of master structures in IpDip by 2PP-DLW

(a), making the negative tone of the master (stamp) onto a UV resist (b)-(d) and replicating the master structure into a thermoplastic by thermal NIL (e)-(f). Modified and reproduced by permission. @ 2016 AVS

\subsection{Post-processing by VUV-exposure and smoothening}

The PMMA patterns were exposed for $30 \mathrm{~s}$ with a VUV intensity of about $16.3 \mathrm{~mW} / \mathrm{cm}^{2}$, at $172 \mathrm{~nm}$ wavelength in a flood exposure tool (EX-mini, Hamamatsu Corp.). After exposure, the lenses were annealed in a top-heater setup (see Fig. 2) at a temperature of $140^{\circ} \mathrm{C}$ for $15 \mathrm{~min}$. This setup allows generating a temperature gradient from the top of the lenses towards the base which additionally supports the concept of surface limited softening. After reflow, the samples were immediately cooled to room temperature, coated with 5-10 $\mathrm{nm}$ chromium and inspected in a scanning electron microscope (SEM, Zeiss Supra 55 VP, Zeiss Microscopy).

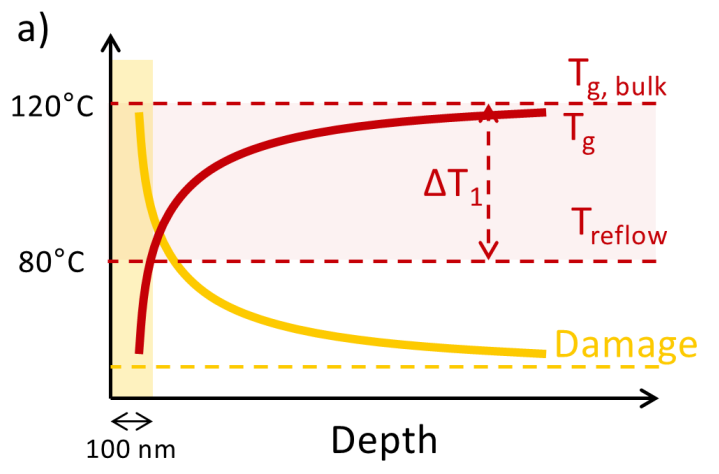

Damage: $M_{w}$ reduction (no ablation) b) $\Delta \mathrm{T}_{1}=\mathrm{T}_{\mathrm{g}, \text { bulk }}-\mathrm{T}_{\text {reflow(100nm) }}$ $\Delta \mathrm{T}_{2}=\mathrm{T}_{\text {bottom }}-\mathrm{T}_{\text {top }}$

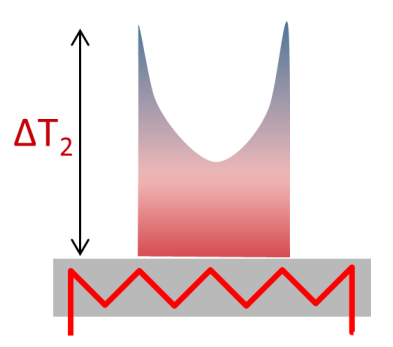

Hot plate (bottom-heater)

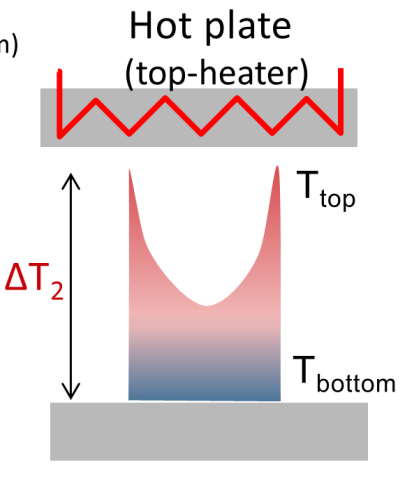

Hot plate

Figure 2. Typical process latitude $\Delta \mathrm{T}_{1}-\Delta \mathrm{T}_{2}$ for the thermal reflow process: a) Balancing glass transition temperature of the bulk $\mathrm{T}_{\mathrm{g}, \text { bulk }}$ of $120^{\circ} \mathrm{C}$ and the process temperature $\mathrm{T}_{\text {reflow }}$. For a reflow of a surface skin layer down to $100 \mathrm{~nm}$ depth only, the exemplary glass temperature $T_{\mathrm{g}, 100 \mathrm{~nm}}$ at this depth has to be about $40 \mathrm{~K}$ smaller than $\mathrm{T}_{\mathrm{g} \text {,bulk }}$ and thus about $80^{\circ} \mathrm{C}$. b) The top-heater setup takes into account small differences in temperature between the top and the bottom of the polymer structures and enables better control of the process temperature. 


\section{RESULTS AND DISCUSSION}

\subsection{Replication into thermoplastic material}

The original IpDip pattern was faithfully replicated into PMMA including the step-to-step roughness due to the writing trajectories used for model discretization (Figure 3). The PMMA replication had a relatively thick residual film which supported together with the flexible PDMS mold the defect free release of the microstructures, the high-aspect ratio features of it as well as the nanotexture on its surface. As found in a previous study [9], the PDMS allowed for a very broad process window $\left(0.5-5 \mathrm{MPa}\right.$ imprint pressure, $140-220^{\circ} \mathrm{C}$ imprint temperature, $1 \mathrm{~s}$ to $180 \mathrm{~min}$ pressure holding time) which makes the replication process very appealing as industrial process. The total writing time for the $3 \times 3$ array was about $5 \mathrm{~min}$ which translates into a writing time for a device with about $2.4 \mathrm{~mm}$ MLA diameter of about $48 \mathrm{~h}$. This might be reduced by further optimization but is already acceptable considering that this is used to make a master structure for further high volume replication processes.
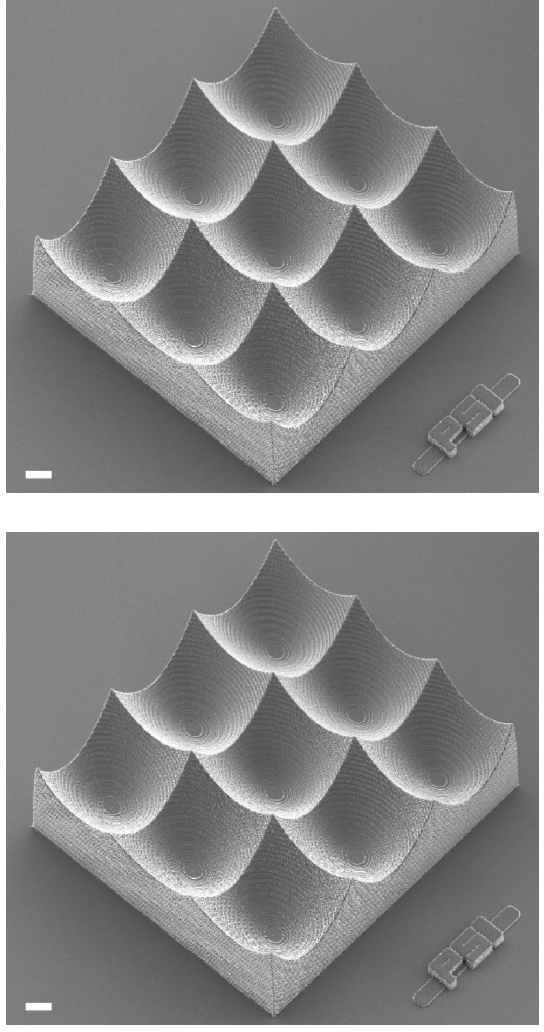

(a)
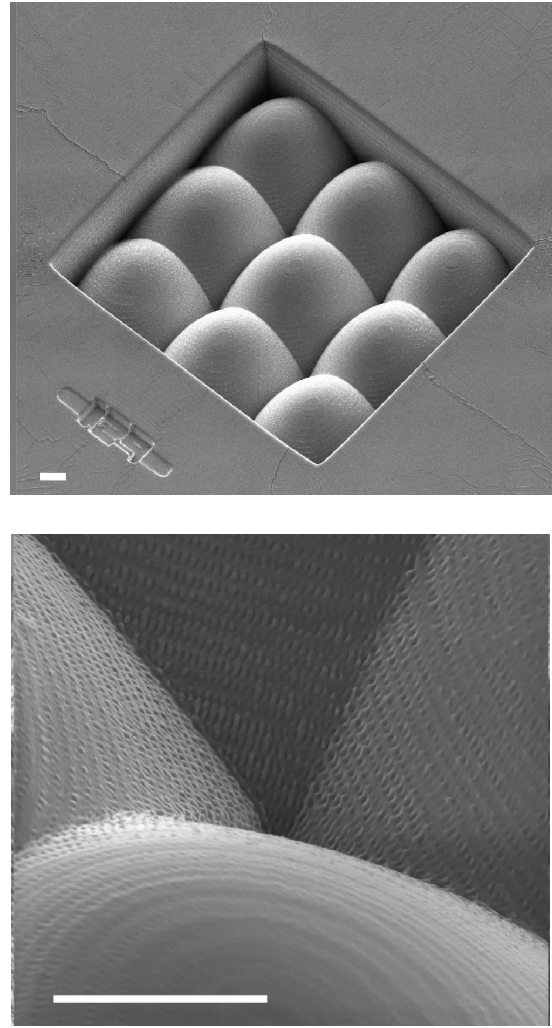

(b)
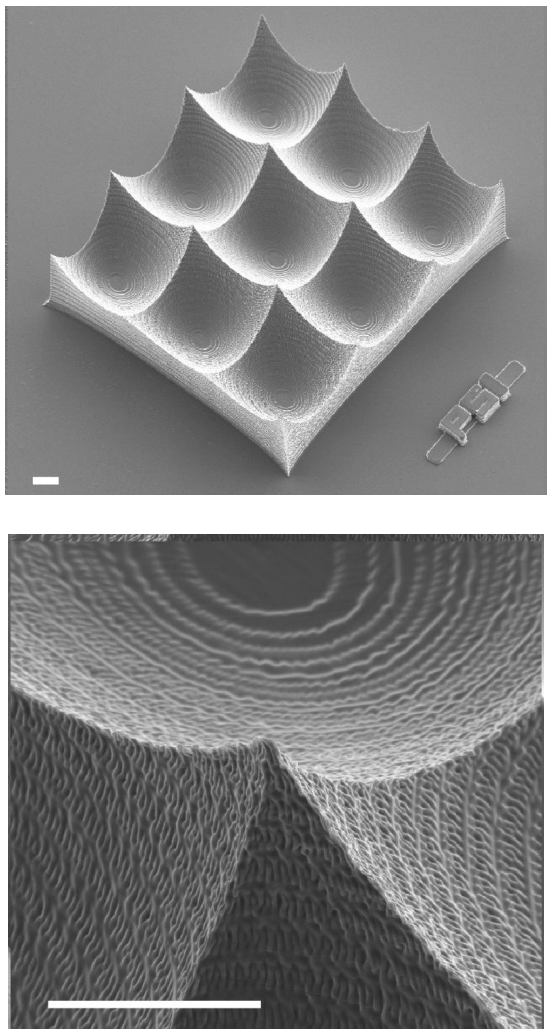

(c)

Figure 3. SEM images of a 3x3 MLA replicated into PMMA to give the same tone as it was written by 2PP-DLW: (a) IpDip resist, (b) UV-PDMS replica and (c) PMMA replica (overview and close-up) with a significant visible roughness due to coarse writing trajectories. The original structure is a microlens array consisting of $3 \times 3$ identical concave lenses with $50 \mu \mathrm{m}$ lateral footprint with $40 \mu \mathrm{m}$ deep central sags surrounded by high aspect ratio tips between adjacent lenses. (scale bar 10 $\mu \mathrm{m})$. Modified and reproduced by permission. @ 2016 AVS

\subsection{Post-processing by VUV exposure and reflow}

VUV radiation is strongly absorbed in PMMA with a linear absorption coefficient of about $5 \mu \mathrm{m}^{-1}$ based on literature values $[20,21]$. This means the relative VUV intensity drops to $1 / \mathrm{e}$ only about $200 \mathrm{~nm}$ below the PMMA surface. This is the region were most of the chain-scission and molecular weight reduction will occur. The molecular weight 
significantly influences the glass transition temperature below a certain critical molecular weight which is $11-15 \mathrm{~kg} / \mathrm{mol}$ for narrow molecular weight distributed PMMA [22]. Measurements and comparison with electron beam exposed PMMA indicate that the molecular weight in the top exposed region is below $10 \mathrm{~kg} / \mathrm{mol}$ [14]. The lower the molecular weight, the lower is the glass transition temperature. First experiments based on the change of the coefficient of thermal expansion and white light reflectometric monitoring of the film thickness during a temperature ramp indicate that the glass transition temperature is below $50^{\circ} \mathrm{C}$ for the top of the VUV exposed PMMA. The glass transition temperature of the unexposed PMMA is about $120^{\circ} \mathrm{C}$ with a weight averaged molecular weight of about $100 \mathrm{~kg} / \mathrm{mol}$ [14]. This means the mobility of the polymer chains on the top surface during the $140^{\circ} \mathrm{C}$ reflow process is significantly higher than that of the bulk material. In addition, the top heating setup further reduces the temperature at the base of the MLA avoiding a feature collapse and thus maintains the structural fidelity. It has to be mentioned that the material displacement or reflow is also dependent on the structure size. A much smaller structure would undergo a significant reflow at the used conditions. However, the micron-sized bulk geometry is not altered visibly.

The post-processing by VUV exposure and thermal reflow completely removed the surface roughness from the PMMA as obtained after replication and gave the surface an ultra-smooth, optical grade finish (Figure 4). This surface smoothening does not significantly alter the overall structural fidelity. The sidewalls of the $3 \times 3$ MLA is basically the same as in Figure 3c. It even still features the same roughness from the writing trajectories. This is most probably due to the limited ability of the material for vertical displacement. Also the high aspect ratio tips are well preserved and thus the radius of the lens curvature is maintained. This will keep the required optical diffusing functionality while homogeneity of the illumination area is improved due to less scattering from surface roughness.

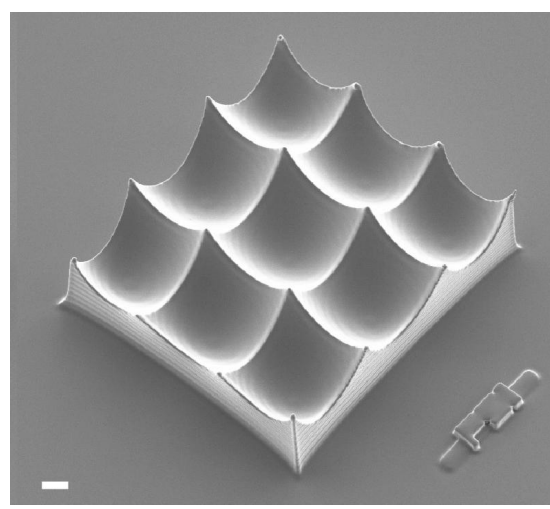

(a)

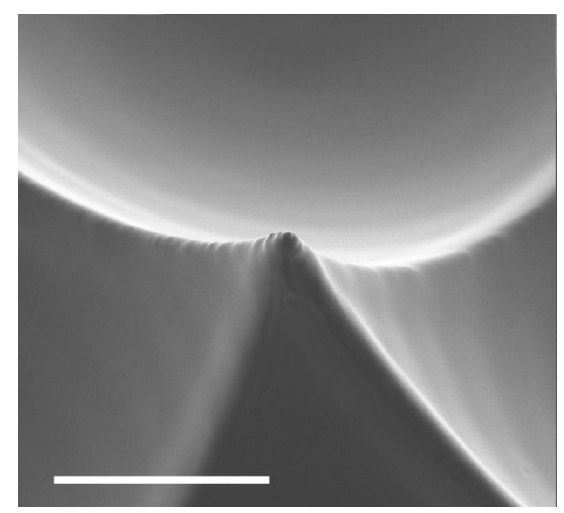

(b)

Figure 4. SEM images of the 3x3 MLA after VUV exposure and thermal reflow: (a) overview and (b) closeup of an intersection between adjacent lens elements. (scale bar $10 \mu \mathrm{m}$ )

The same replication and smoothening process can be also applied to other micro-optical structures such as a $5 \times 5$ microprism array (Figure 5). This demonstrates the versatility of the post-processing concept. For an optimal writing time and smooth surfaces by direct writing, the different angles in the microprism array would require different 2PPDLW discretization levels or writing strategies. In contrast, the post-processing, as described in our work here, is geometry independent. As with the MLA, the structural fidelity is maintained during the reflow for the prism array and the sharp transition with steep sidewalls between adjacent microprism facets are unaltered. 

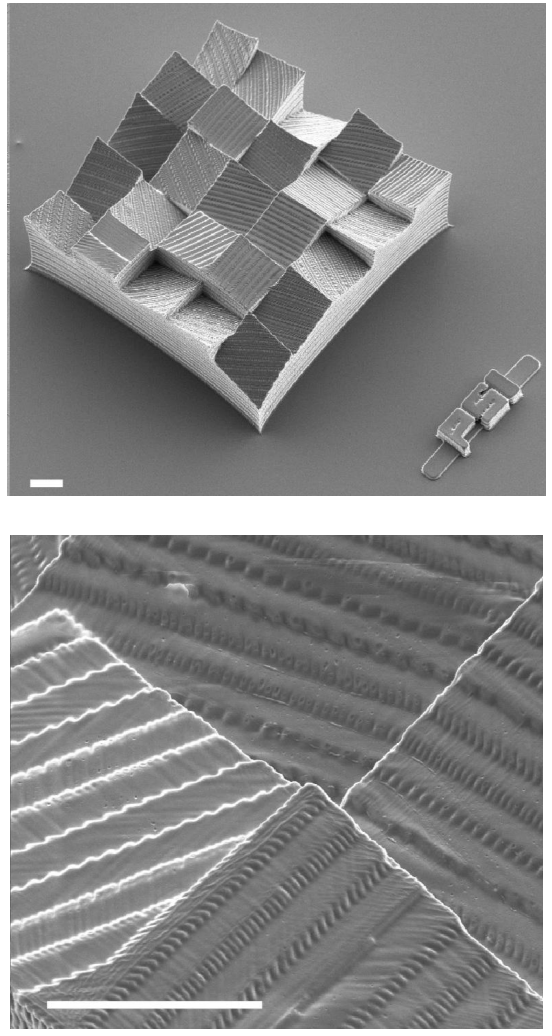

(a)
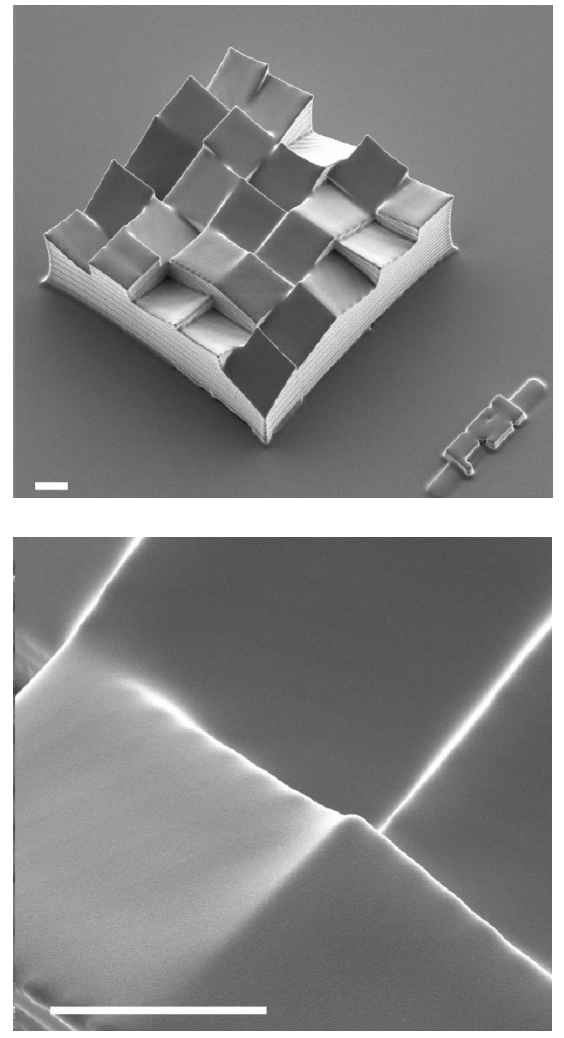

(b)

Figure 5. SEM images of a 5×5 microprism array (a) following PMMA replication and (b) after VUV exposure and thermal reflow. The original structure is consisting of tiles with $20 \mu \mathrm{m}$ lateral footprint and different inclination angles (overview and close-up: scale bar $10 \mu \mathrm{m}$ )

\section{CONCLUSION}

We demonstrated a post-processing method that can be applied to remove the inherent writing roughness of 2PP-DLW from micro-optics. This approach clearly addresses fast prototyping and mastering of micro-optics and can be applied to various designs and materials. The smoothening process only involves a material relocation in the top 100-500 nm and does not involve a bulk material softening below this top surface. This allows using rather coarse 2PP discretization in the range of 300-400 $\mathrm{nm}$ vertical step height and thus a fast writing process. The surface confined smoothening is also useful for other linear thermoplastics that undergo a chain-scission and molecular weight reduction due to high energy photon impact. In contrast, radiation hardening materials such as polystyrene or negative tone resists like SU-8 might be used to create a hard surface on top of a less hard core.

\section{ACKNOWLEDGEMENT}

This project was funded by the Swiss Nanoscience Institute (project A10.13 SurfFlow). The authors express their special thanks to K. Vogelsang (PSI) for support with the hot embossing. The authors also thank M. Rossi (Heptagon) for the support of this project and the useful discussion. 


\section{REFERENCES}

[1] Schift, H., Urwyler, P., Kristiansen, P.M., and Gobrecht, J., "Nanoimprint lithography process chains for the fabrication of micro- and nanodevices," J. Micro/Nanolith. MEMS MOEMS 13(3), 031303 (2014)

[2] Gale, M.T., Rossi, M., Schuetz, H., "Fabrication of continuous-relief micro-optical elements by direct laser writing in photoresist, laser-assisted fabrication of thin films and microstructures," Proc. SPIE 2045, 54-62 (1994)

[3] Schift, H., Söchtig, J., Rossi, M., and Westenhöfer, S., "Replicated high precision micro-optical insert elements for optical bench arrangements," Proc. SPIE 3513, 122-134 (1998)

[4] Heckele, M., Schomburg W.K., "Review on micro molding of thermoplastic polymers," J. Micromech. Microeng., 14, R1 (2004)

[5] Stäger, B., Gale, M.T. and Rossi, M., "Replicated micro-optics for automotive applications," Proc. SPIE 5663, 238-245 (2005)

[6] Becker, E.W., Ehrfeld, W., Hagmann, P., Maner, A., Münchmeyer D., "Fabrication of microstructures with high aspect ratios and great structural heights by synchrotron radiation lithography, galvanoforming, and plastic moulding (LIGA process)," Microelectron. Eng. 4 (1), 35-56 (1986)

[7] Saile, V., Wallrabe, U., Tabata, O., [LIGA and its Applications], in Advanced Micro and Nanosystems (Vol. 7), series eds. J.G. Korvink, O. Brand, G.K. Fedder, C. Hierold, 1st edn. Wiley-VCH, Weinheim (2008)

[8] Cadarso, V.J., Chidambaram, N., Jacot-Descombes, L., and Schift, H., "High aspect ratio nanoimprint process chains," accepted for publication in nature microsystems \& nanoengineering (2017)

[9] Chidambaram, N., Kirchner, R., Altana, M. and Schift, H., "High fidelity 3D thermal nanoimprint with UV curable polydimethyl siloxane stamps," J. Vac. Sci. Technol. B 34, 06K401 (6pp) (2016)

[10] Schift, H., "Nanoimprint lithography, 2D or not 2D? A review," Appl. Phys. A 21 (2), 415-435 (2015).

[11] Gissibl, T., Thiele, S., Herkommer, A. and Giessen, H. "Two-photon direct laser writing of ultracompact multilens objectives," Nat. Phot. 10, 554-561 (2016)

[12] Kirchner, R. Chidambaram, N., Altana, M., and Schift, H., "How post-processing by selective thermal reflow can reduce the roughness of 3D lithography in micro-optical lenses," Proc. SPIE 10095-5 (2017)

[13] Schleunitz, A. and Schift, H., "Fabrication of 3-D nanoimprint stamps with continuous reliefs using dosemodulated electron beam lithography and thermal reflow," J. Micromech. Microeng. 20, 095002 (2010)

[14] Schleunitz, A., Guzenko, V. A., Messerschmidt, M., Atasoy, H., Kirchner, R. and Schift, H., "Novel 3D microand nanofabrication method using thermally activated selective topography equilibration (TASTE) of polymers," Nano Converg. 1:7, 1-8 (2014)

[15] Kirchner, R., Schleunitz, A., and Schift, H., "Energy-based thermal reflow simulation for 3D polymer shape prediction using the surface evolver," J. Micromech. Microeng. 24 (5), 055010 (7pp) (2014)

[16] Kirchner, R. and Schift, H., "Mobility based 3D simulation of selective, viscoelastic polymer reflow using Surface Evolver," J. Vac. Sci. Technol. 32 (6), 06F701 (7pp.) (2014)

[17] Chidambaram, N., Kirchner, R. Fallica, R., Yu, L., Altana, M., Schift, H., "Selective surface smoothening of polymer microlenses by depth confined softening," submitted (2017).

[18] Schift, H., "Nanoimprint lithography: An old story in modern times? A review," J. Vac. Sci. Technol. B 26 (2), 458-480 (2008)

[19] Schift, H., Saxer, S., Park, S., Padeste, C., Pieles, U., and Gobrecht, J., "Controlled co-evaporation of silanes for nanoimprint stamps," Nanotechnology 16, S171-S175 (2005)

[20] Fouchier, M., Pargon, E., Azarnouche, L., Menguelti, K., Joubert, O., Cardolaccia, T. and Bae, Y. C., "Vacuum ultra violet absorption spectroscopy of 193 nm photoresists," Appl. Phys. A 105(2), 399-405 (2011)

[21] Philipp, H. R., Cole, H. S., Liu, Y. S. and Sitnik, T. A., "Optical absorption of some polymers in the region 240170 nm," Appl. Phys. Lett. 48 (2), 192-194 (1986)

[22]Fuchs, K., Friedrich, C. and Weese, J., "Viscoelastic properties of narrow-distribution poly(methyl methacrylates)," Macromolecules 29, 5893-5901 (1996) 